\title{
The emergence of the first star-free atomic cooling haloes in the Universe
}

\author{
John A. Regan ${ }^{\oplus},{ }^{1 \star}$ John H. Wise, ${ }^{2}$ Brian W. O’Shea ${ }^{3,4,5,6}$ and Michael L. Norman ${ }^{7}$ \\ ${ }^{1}$ Centre for Astrophysics and Relativity, School of Mathematical Sciences, Dublin City University, Glasnevin D09 W6Y4, Ireland \\ ${ }^{2}$ Center for Relativistic Astrophysics, Georgia Institute of Technology, 837 State Street, Atlanta, GA 30332, USA \\ ${ }^{3}$ National Superconducting Cyclotron Laboratory, Michigan State University, 640 S Shaw Ln, East Lansing, MI 48823, USA \\ ${ }^{4}$ Department of Physics and Astronomy, Michigan State University, 567 Wilson Rd, East Lansing, MI 48823, USA \\ ${ }^{5}$ Department of Computational Mathematics, Science and Engineering, Michigan State University, 428 S Shaw Ln, East Lansing, MI 48823, USA \\ ${ }^{6}$ Joint Institute for Nuclear Astrophysics - Center for the Evolution of the Elements, 640 S Shaw Lane, East Lansing, MI 48824, USA \\ ${ }^{7}$ Center for Astrophysics and Space Sciences, University of California, San Diego, 9500 Gilman Dr, La Jolla, CA 92093, USA
}

Accepted 2019 December 28. Received 2019 October 30; in original form 2019 August 7

\begin{abstract}
Using the Renaissance suite of simulations, we examine the emergence of pristine atomic cooling haloes that are both metal free and star free in the early universe. The absence of metals prevents catastrophic cooling, suppresses fragmentation, and may allow for the formation of massive black hole seeds. Here we report on the abundance of pristine atomic cooling haloes found and on the specific physical conditions that allow for the formation of these directcollapse-black hole (DCBH) haloes. In total, in our simulations we find that 79 DCBH haloes form before a redshift of 11.6. We find that the formation of pristine atomic haloes is driven by the rapid assembly of the atomic cooling haloes with mergers, both minor and/or major, prior to reaching the atomic cooling limit a requirement. However, the ability of assembling haloes to remain free of (external) metal enrichment is equally important and underlines the necessity of following the transport of metals in such simulations. The candidate DCBH-hosting haloes we find have been exposed to mean Lyman-Werner radiation fields of $J_{21} \sim 1$ and typically lie at least $10 \mathrm{kpc}$ (physical) from the nearest massive galaxy. The growth rates of the haloes reach values of greater than $10^{7} \mathrm{M}_{\odot}$ per unit redshift, leading to significant dynamical heating and the suppression of efficient cooling until the halo crosses the atomic cooling threshold. Finally, we also find five synchronized halo candidates where pairs of pristine atomic cooling haloes emerge that are both spatially and temporally synchronized.
\end{abstract}

Key words: methods: numerical-stars: black holes-large-scale structure of Universecosmology: theory.

\section{INTRODUCTION}

Supermassive black holes (SMBHs) with masses upwards of one billion solar masses have been observed less than one billion years after the big bang (Fan, Carilli \& Keating 2006; Mortlock et al. 2011; Wu et al. 2015; Bañados et al. 2018). However, the mechanisms that allow for the formation of SMBHs are hotly debated and currently unknown (for a recent review, see Woods et al. 2018). The mainstream scenarios fall into two main brackets. The first mechanism uses light seeds as the origin for the massive black hole seeds. Light seeds are thought to have masses between 30 and 1000 $\mathrm{M}_{\odot}$ masses and may be formed from the end point of Population III (PopIII) stars (Madau \& Rees 2001; Abel, Bryan \& Norman 2002; Bromm, Coppi \& Larson 2002). Light seeds may also evolve from the core collapse of a dense stellar cluster (Begelman \& Rees 1978; Freitag, Gürkan \& Rasio 2006; Freitag 2008; Devecchi \&

^E-mail: john.regan@mu.ie
Volonteri 2009; Merritt 2009; Lupi et al. 2014; Katz, Sijacki \& Haehnelt 2015) where stellar collisions result in the formation of a massive black hole. However, there is a general consensus within the community that growing from light seed masses up to one billion solar masses may be demanding in the early Universe and that the vast majority of light seeds suffer from starvation in their host halo (Whalen, Abel \& Norman 2004; Alvarez, Wise \& Abel 2009; Milosavljević, Couch \& Bromm 2009; Smith et al. 2018); however, see Alexander \& Natarajan (2014), Inayoshi, Haiman \& Ostriker (2016), and Pacucci et al. (2017) for examples of superEddington accretion mechanisms that may circumvent light seed growth restrictions.

The second mechanism advocates for heavy seeds with initial masses between 1000 and $100000 \mathrm{M}_{\odot}$. This scenario is commonly referred to as the 'direct-collapse black hole' (DCBH) scenario (Eisenstein \& Loeb 1995; Oh \& Haiman 2002; Bromm \& Loeb 2003) and relies on the direct collapse of a metal-free gas cloud directly into a massive black hole. Depending on the exact thermodynamic conditions of the collapse, the massive black hole phase 
may be preceded by an intermediary stage involving a supermassive star (Shapiro \& Teukolsky 1979; Hosokawa et al. 2013; Schleicher et al. 2013; Inayoshi, Omukai \& Tasker 2014; Woods et al. 2017; Haemmerlé et al. 2018a, b) or a quasi-star (Begelman, Volonteri \& Rees 2006; Begelman, Rossi \& Armitage 2008). Initial numerical investigations of the collapse of atomic cooling haloes revealed that the collapse could proceed monolithically and that the formation of a massive black hole seed with a mass up to $100000 \mathrm{M}_{\odot}$ masses was viable in the early Universe where atomic cooling haloes were both metal free and free of $\mathrm{H}_{2}$ (Bromm et al. 2002; Wise, Turk \& Abel 2008; Regan \& Haehnelt 2009a, b).

As the numerical investigations became more sophisticated, the research landscape shifted to understanding how metal-free atomic cooling haloes could exist, which remained free of rampant star formation. $\mathrm{H}_{2}$ cooling within minihaloes, which would precede atomic cooling haloes, would lead to the formation of PopIII stars, thus shutting off the pathway to massive black hole seed formation. $\mathrm{H}_{2}$ can be dissociated by radiation in the Lyman-Werner (LW) band (Field, Somerville \& Dressler 1966) between 11.8 and $13.6 \mathrm{eV}$. If the intensity of $\mathrm{LW}$ radiation is strong enough, then $\mathrm{H}_{2}$ formation can be suppressed, allowing for the formation of an atomic cooling halo in which $\mathrm{H}_{2}$ cooling is prevented and the halo must cool and collapse on the so-called atomic track. A number of authors (Shang, Bryan \& Haiman 2010; Wolcott-Green, Haiman \& Bryan 2011; WolcottGreen \& Haiman 2012; Regan, Johansson \& Haehnelt 2014a; Sugimura, Omukai \& Inoue 2014; Visbal, Haiman \& Bryan 2014a; Agarwal \& Khochfar 2015; Latif et al. 2015) examined the intensity of $\mathrm{LW}$ radiation required to completely suppress $\mathrm{H}_{2}$ formation and found that the intensity of LW radiation impinging on to a nascent halo needed to be upwards of $1000 \mathrm{~J}_{21} .{ }^{1}$ Only pristine and metalfree haloes in close proximity to another rapidly star-forming halo would be able to fulfil that criterion given that the $1000 \mathrm{~J}_{21}$ value is orders of magnitude above expected mean background values (e.g. Ahn et al. 2009). Two haloes developing closely, separated in both time and space, would allow for this mechanism and hence the 'synchronized-halo' model was developed by Dijkstra et al. (2008), which advocated this approach as being conducive to the formation of atomic cooling haloes that allow the full suppression of $\mathrm{H}_{2}$. Regan et al. (2017) tested the theory rigorously through numerical simulations, showing that atomic cooling haloes that develop and are subhaloes of one another can lead to the complete suppression of $\mathrm{H}_{2}$ in one of the haloes and hence to an isothermal collapse of the core of one of the pairs. The exact abundance of synchronized haloes is challenging to predict analytically and even in optimistic evaluations the number density of synchronized pairs may only be able to seed a subpopulation of all SMBHs (Visbal, Haiman \& Bryan 2014b; Inayoshi \& Tanaka 2015; Habouzit et al. 2016).

More recently, Wise et al. (2019), hereafter W19, showed that the rapid assembly of haloes can also lead to the suppression of $\mathrm{H}_{2}$ and should be significantly more common than the synchronized pair scenario (though this mechanism does not necessarily lead to a pure isothermal collapse while the synchronized scenario should). Dynamical heating (Yoshida et al. 2003; Fernandez et al. 2014) can suppress the impact of $\mathrm{H}_{2}$ cooling, thus keeping an assembling halo hotter and preventing the formation of stars. W19 investigated two haloes in particular from a set of high-resolution adaptive mesh refinement simulations of the early Universe that they found had breached the atomic cooling limit, were metal free, and had

${ }^{1} J_{21}$ is shorthand for $1 \times 10^{-21} \mathrm{erg} \mathrm{s}^{-1} \mathrm{~cm}^{-2} \mathrm{~Hz}^{-1} \mathrm{sr}^{-1}$ and measures the intensity of radiation at a given point. not formed stars. The two haloes that they targeted for detailed examination were the most massive halo (MMHalo) and the most irradiated halo (LWHalo) at the final output of the simulation, redshift 15 . W19 found that the haloes were subject to only relatively mild LW exposure and that in the absence of all other external effects should have formed stars. They found that the haloes experienced especially rapid growth compared to typical haloes and that the extra dynamical heating effects driven by the rapid growth allowed the haloes to remain star free. Their examinations also showed that the haloes did not show any initial signs of rapid collapse - however, they did not run their simulations beyond the formation of the first density peak, and further evolution of these haloes is still required to determine the detailed characteristics of the objects that form. In this study, we examine the entire data set of metal-free and starfree haloes produced by the simulations used in W19. As such, this study is more comprehensive and allows for a broader analysis of the physics driving the formation of these pristine objects. The goal of this study is to look at the Renaissance simulation data set in its entirety. Here we identify DCBH candidates at each redshift and also investigate the environmental conditions that lead to the emergence of atomic cooling haloes that are both metal free and star free.

\section{RENAISSANCE SIMULATION SUITE}

The Renaissance simulations were carried out on the Blue Waters supercomputer facility using the adaptive mesh refinement code Enzo (Bryan et al. 2014). ${ }^{2}$ Enzo has been extensively used to study the formation of structure in the early universe (Abel et al. 2002; O'Shea et al. 2005; Turk et al. 2012; Wise et al. 2012, 2014; Regan, Johansson \& Wise 2015; Regan et al. 2017). In particular, Enzo includes a ray-tracing scheme to follow the propagation of radiation from star formation and black hole formation (Wise \& Abel 2011) as well as a detailed multispecies chemistry model that tracks the formation and evolution of nine species (Abel et al. 1997; Anninos et al. 1997). In particular, the photodissociation of $\mathrm{H}_{2}$ is followed, which is a critical ingredient for determining the formation of the first metal-free stars (Abel, Bryan \& Norman 2000).

The data sets used in this study were originally derived from a simulation of the universe in a $28.4 h^{-1} \mathrm{Mpc}$ on the side box using the WMAP7 best-fitting cosmology. Initial conditions were generated using MUSIC (Hahn \& Abel 2011) at $z=99$. A low-resolution simulation was run until $z=6$ in order to identify three different regions for re-simulation (Chen et al. 2014). The volume was then smoothed on a physical scale of 5 comoving Mpc, and regions of high $\left[\langle\delta\rangle \equiv\langle\rho /\rangle\left(\Omega_{\mathrm{M}} \rho_{\mathrm{C}}\right)-1 \simeq 0.68\right]$, average $[\langle\delta\rangle \sim 0.09]$, and low $[\langle\delta\rangle \simeq-0.26]$ mean density were chosen for re-simulation. These subvolumes were then referred to as the Rarepeak region, the Normal region, and the Void region. The Rarepeak region has a comoving volume of $133.6 \mathrm{Mpc}^{3}$, and the Normal region and the Void regions have comoving volumes of $220.5 \mathrm{Mpc}^{3}$. Each region was then re-simulated with an effective initial resolution of $4096^{3}$ grid cells and particles within these subvolumes of the larger initial simulation. This gives a maximum dark matter particle mass resolution of $2.9 \times 10^{4} \mathrm{M}_{\odot}$. For the re-simulations of the Void, Normal, and Rarepeak regions, further refinement was allowed throughout the subvolumes up to a maximum refinement level of 12 , which corresponded to a 19 pc comoving spatial resolution. Given that the regions focus on different overdensities, each region was evolved forward in time to different epochs. The Rarepeak region,

\footnotetext{
${ }^{2}$ https://enzo-project.org/
} 

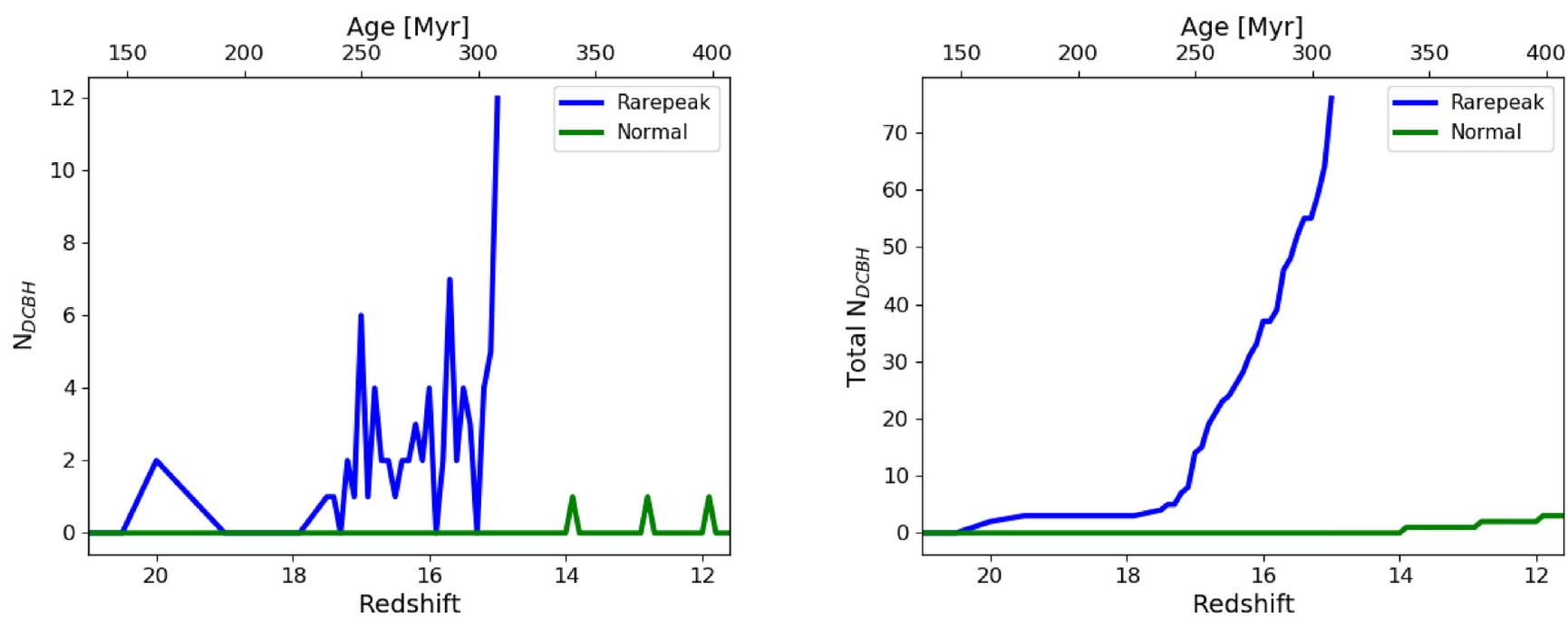

Figure 1. Left-hand panel: The number of DCBH candidate haloes found at each redshift in each region. Right-hand panel: The total number of DCBH candidate haloes found as a function of redshift. The Rarepeak region (blue line) has formed a total of 76 candidate DCBH haloes. The Normal region (green line) has formed a total of three DCBH candidate haloes. The running total is the total number of DCBH candidate haloes formed over the entire simulation once duplicates are excluded and accounting for a DCBH candidate halo becoming subsequently polluted. For completeness, the age of the universe at that time is included at the top of each figure.

being the most overdense and hence the most computationally demanding at earlier times, was run until $z=15$. The Normal region ran until $z=11.6$, and the Void region ran until $z=8$. In all of the regions, the halo mass function was very well resolved down to $M_{\text {halo }} \sim 2 \times 10^{6} \mathrm{M}_{\odot}$. The Rarepeak regions contained 822 galaxies with masses larger than $10^{9} \mathrm{M}_{\odot}$ at $z=15$, the Normal region contained 758 such galaxies at $z=11.6$, and the Void region contained 458 such galaxies at $z=8$.

As noted already in section 1, in W19, we examined two metalfree and star-free haloes from the Rarepeak simulation. Only the $z=15$ data set was used. In this work, we examine all of the data sets available from the Void, Normal, and Rarepeak regions to get a larger sample of the emergence of DCBH haloes across all three simulations and across all redshift outputs. In the next section, we examine both the number density of DCBH across time and also the environmental conditions that lead to their appearance.

\section{RESULTS}

We investigate here the emergence of $\mathrm{DCBH}$ candidate haloes in the Renaissance simulations. We first investigate the absolute number of DCBH candidate haloes that form in each of the three simulation regions. We then examine in more detail the physical conditions that allow their emergence.

\subsection{The abundance of DCBH candidate haloes}

In the left-hand panel of Fig. 1, we show the absolute number of candidate DCBH haloes in each simulation region over the range of redshift outputs available to us. In the right-hand panel, we show the running total for the number of candidate DCBH haloes formed over the course of the entire simulation. As noted in Section 2, the Rarepeak simulation runs to $z=15$, the Normal simulation runs to $z=11.6$ and the Void simulation runs to $z=8$. At each redshift snapshot, we calculate the number of metal-free, atomic cooling haloes that contain no stars. The number of these DCBH candidate haloes, $N_{\mathrm{DCBH}}$, versus redshift is captured in the left-hand panel of Fig. 1. The Rarepeak simulation (blue line) contains the largest absolute number of DCBH candidate haloes. At the final output time $(z=15)$, there are 12 candidate DCBH haloes in the Rarepeak volume. This compares to 0 in the Normal volume at $z$ $=11.6$. However, there are candidates detected in the Normal region at other outputs as we can see. No candidates are detected in the void region at any redshift output and hence we do not explore the Void region any further in this work.

We can see that the number of DCBH candidate haloes fluctuates over time although, overall, the trend is that there is an increase in the number of the DCBH candidate haloes per unit redshift. The increase is more prominently seen in the right-hand panel of Fig. 1. The running total for the number of DCBH candidate haloes increases rapidly and by $z=15$ the Rarepeak simulation has hosted $76 \mathrm{DCBH}$ halo candidates while the Normal region has hosted 3 DCBH halo candidates. The cumulative total accounts for the fact that a previous DCBH candidate halo can become polluted and hence no longer matches the criteria even though it may now host a DCBH. ${ }^{3}$ In contrast, the left-hand panel is a pure snapshot at that time and has no memory of the history of haloes. In Fig. 2, we plot the location of each of the distinct DCBH candidate haloes on top of a projection of the number density of the Rarepeak region and of the Normal region. In each case, the projection is made at the final redshift output (Rarepeak, $z=15$; Normal, $z=11.6$ ). The dashed red circles, which denote the halo location, are from across all redshift outputs and hence should be seen as approximate locations. None the less, what is immediately obvious is that the emergence of DCBH candidate haloes is a ubiquitous feature of high-density regions. The number of haloes in the Normal region is significantly reduced compared to the Rarepeak region. The reason behind the much larger number of DCBH candidates in the Rarepeak region compared to the Normal region is multifaceted, depending on the

\footnotetext{
${ }^{3}$ Renaissance has no subgrid model for DCBH formation and so DCBH is not recorded as haloes assemble.
} 

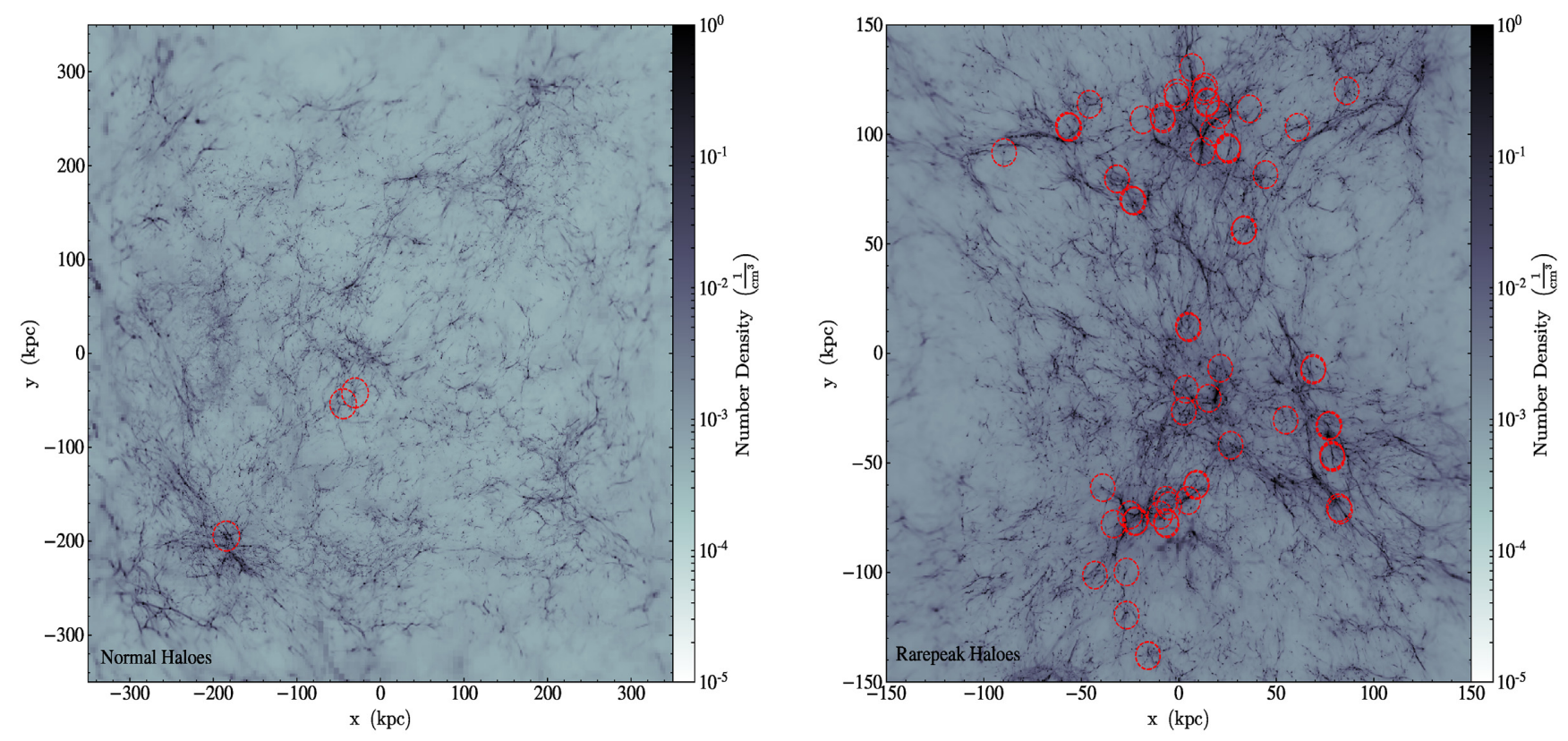

Figure 2. Left-hand panel: Projection of the Normal simulation volume with dashed red circles identifying the location of all three DCBH halo candidates across all redshift outputs. Right-hand panel: Projection of the Rarepeak simulation volume with dashed red circles identifying the location of all 76 DCBH candidates across all redshift outputs. The Rarepeak projection is made at $z=15$ and the Normal projection is made at $z=11.6$ although the DCBH candidate haloes may have formed at a different epoch.

growth of structure, the mean density of the intergalactic medium in that region, and the flux of LW radiation.

The number of galaxies above some given minimum mass $M_{\min }(z)$ in a redshift bin of width $d z$ and solid angle $d \Omega$ can be defined using the Press-Schechter formalism (Press \& Schechter 1974).

$$
\frac{\mathrm{d} M}{\mathrm{~d} \Omega \mathrm{d} z}(z)=\frac{\mathrm{d} V}{\mathrm{~d} \Omega \mathrm{d} z}(z) \int_{M_{\min }(z)}^{\mathrm{inf}} \mathrm{d} M \frac{\mathrm{d} n}{\mathrm{~d} M}(M, z),
$$

where $\mathrm{d} V / \mathrm{d} \Omega \mathrm{d} z$ is the cosmological comoving volume element at a given redshift and $(\mathrm{d} n / \mathrm{d} M) \mathrm{d} M$ is the comoving halo number density as a function of mass and redshift. The latter quantity was expressed by Jenkins et al. (2001) as

$$
\begin{aligned}
\frac{\mathrm{d} n}{\mathrm{~d} M}(M, z)= & -0.315 \frac{\rho_{o}}{M} \frac{1}{\sigma_{M}} \frac{\mathrm{d} \sigma_{M}}{\mathrm{~d} M} \\
& \left.\times \exp \left(-\left|0.61-\log \left(D(z) \sigma_{M}\right)\right|^{3.8}\right)\right),
\end{aligned}
$$

where $\sigma_{M}$ is the RMS density fluctuation, computed on mass scale $M$ from the $z=0$ linear power spectrum (Eisenstein \& Hu 1999); $\rho_{0}$ is the mean matter density of the universe, defined as $\rho_{0}=$ $\Omega_{M} * \rho_{\mathrm{c}}$ (with $\rho_{\mathrm{c}}$ being the cosmological critical density, defined as $\rho_{\mathrm{c}}=3 H_{0}^{2} / 8 \pi G$ ), and $D(z)$ is the linear growth function (see e.g. Hallman et al. 2007, for details). Taking this together, we find that $\mathrm{d} n / \mathrm{d} M$ scales approximately as $\rho \sigma_{M}^{3.8}$.

The higher mean density and higher $\sigma_{M}$ in the Rarepeak compared to the Normal region are therefore consistent with previous findings showing that there are approximately 3-4 times more haloes, per unit redshift, in the Rarepeak region (Xu, Wise \& Norman 2013; O'Shea et al. 2015). Not only this, the higher mean densities in the Rarepeak region lead to a smaller volume-filling fraction of metal enrichment in the Rarepeak region compared to the Normal region. Taking supernova blastwave calculations alone leads to a volume-filling fraction of 0.7 in the Rarepeak relative to the Normal region. Finally, the flux of LW is also much higher in the Rarepeak region as there are more haloes producing more stars per unit volume compared to the Normal region (see e.g. Xu et al. 2013). The combination of these three factors leads to significantly more DCBH candidate haloes in the Rarepeak region. Over the time interval that the Renaissance simulations run for, this leads to a ratio of 76 DCBH candidates in the Rarepeak region compared to just 3 in the Normal region.

\subsection{The physical conditions required for DCBH candidate halo formation}

In Fig. 3, we plot the distance from each DCBH candidate halo to the nearest massive galaxy and we also plot the level of LW radiation that each candidate halo is exposed to. In the left-hand panel of Fig. 3, the distance ${ }^{4}$ to the nearest massive galaxy (defined below) is calculated by examining every halo in a sphere of radius $1 \mathrm{Mpc}$ around the DCBH candidate halo. The stellar mass in each halo is then normalized by the square of the distance between that halo and the candidate halo. This normalization accounts for the $r^{2}$ dropoff in radiation intensity with distance. The galaxy with the largest normalized stellar mass is then used as the nearest massive galaxy. In the Rarepeak simulation, most galaxies lie at least $10 \mathrm{kpc}$ away but the spread is quite even up to nearly $100 \mathrm{kpc}$ at which point it starts to decline. In the Normal simulation, which only has three candidates, the nearby galaxies lie approximately 5 and $50 \mathrm{kpc}$ (in two of the cases) away. What this tells us is that close proximity to nearby starforming galaxies is not (directly) correlated with forming DCBH candidate haloes. In the right-hand panel, we investigate the level of $\mathrm{LW}$ radiation that each candidate halo is exposed to at the associated redshift output. In this case, the results are somewhat more defined. For the Rarepeak region, the values of $J_{\mathrm{LW}}$ are between 0.01 and

\footnotetext{
${ }^{4}$ All distances discussed are in physical units unless explicitly stated otherwise.
} 

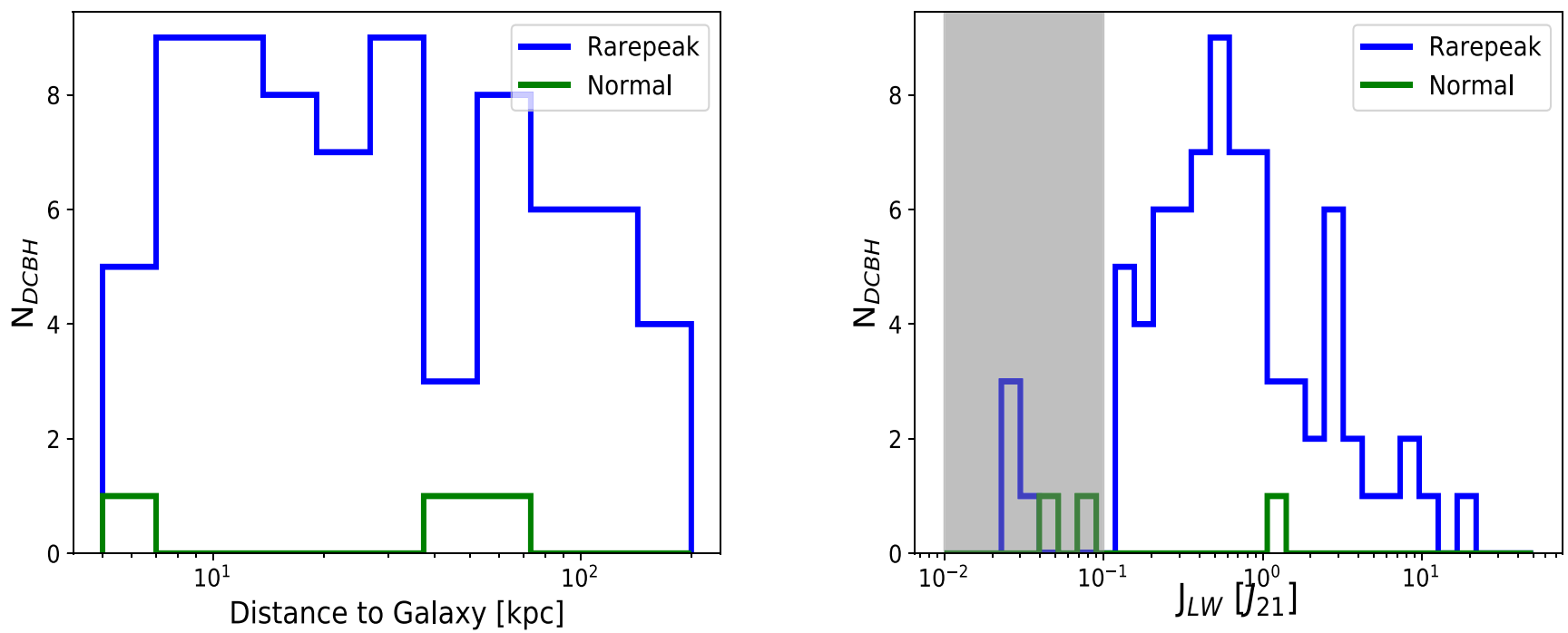

Figure 3. Left-hand panel: The distance from each candidate DCBH halo to the nearest massive galaxy (defined as the closest star-forming halo; see the text for more details) for each region. Right-hand panel: The value of the LW background, in units of $\mathrm{J}_{21}$, felt at the centre of each DCBH candidate. For the majority of DCBH haloes, the value of LW radiation it is exposed to is within an order of magnitude of the background level at that redshift. Only a small number of DCBH candidate haloes experience radiation levels more than one order of magnitude higher than the background level. The grey vertical band indicates the approximate level of background LW radiation expected at $z=15$ (Ahn et al. 2009; Xu et al. 2013).
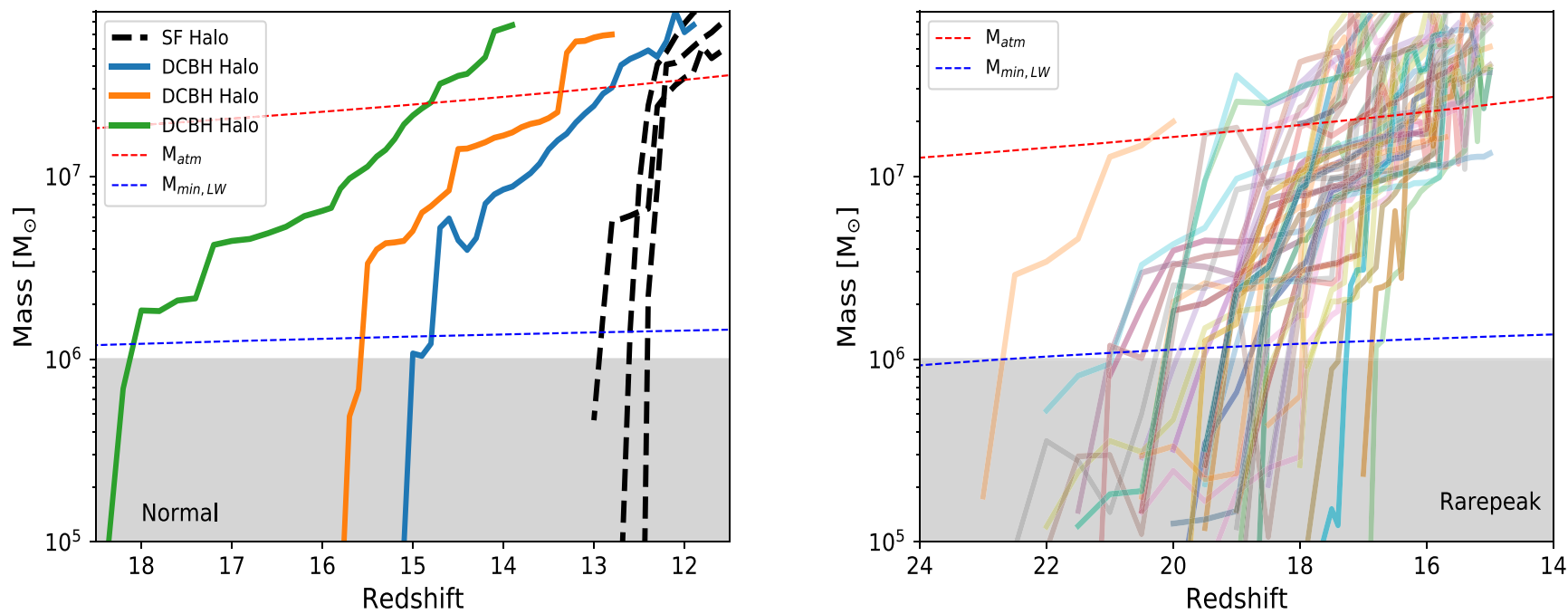

Figure 4. Left-hand panel: The evolution of the total mass of each DCBH candidate halo in the Normal simulation. Also included (dashed black lines) is the evolution of three rapidly growing star-forming haloes for comparison. The mass resolution of the Renaissance simulations is approximately $20000 \mathrm{M}_{\odot}$, so values below $10^{6} \mathrm{M}_{\odot}$ should be treated with caution and we therefore set the halo resolution of our analysis at $10^{6} \mathrm{M}_{\odot}$. Right-hand panel: The evolution of the total mass of each DCBH candidate halo in the Rarepeak simulation. In the vast majority of cases, the halo grows rapidly just prior to reaching the atomic cooling limit.

$10 \mathrm{~J}_{21}$, while for the Normal simulation the values are between approximately 0.1 and $1 \mathrm{~J}_{21}$, albeit for significantly fewer DCBH candidate haloes. The values for the LW radiation field, in the Rarepeak region, are approximately an order of magnitude higher than the expected mean radiation field at this redshift of $J_{\mathrm{LW}}=10^{-2}-$ $10^{-1} \mathbf{J}_{21}$ (Ahn et al. 2009; Xu et al. 2013) - marked by the shaded region in Fig. 3. The reason for this is that the Rarepeak region has significantly more galaxies (O'Shea et al. 2015) compared to the Normal region and the galaxies are also much brighter, especially in the LW band.

The level of LW radiation felt by the vast majority of candidate DCBH haloes is significantly below the level required to fully suppress $\mathrm{H}_{2}$ cooling (Latif et al. 2014; Regan, Johansson \& Wise 2014b; Regan, Johansson \& Wise 2016), which is typically estimated to be approximately $1000 \mathrm{~J}_{21}$. None the less, the haloes do not collapse until after reaching the atomic cooling limit. As we found in W19, the impact of rapid halo growth plays a dominant role in the halo assembly history of these haloes, as we now discuss.

In Fig. 4, we plot the mass growth of each candidate DCBH halo as a function of redshift. In both the panels, we plot the mass of the halo versus the redshift. The left-hand panel contains haloes from the Normal simulation while the right-hand panel contains haloes from the Rarepeak simulation. The grey region in each panel 

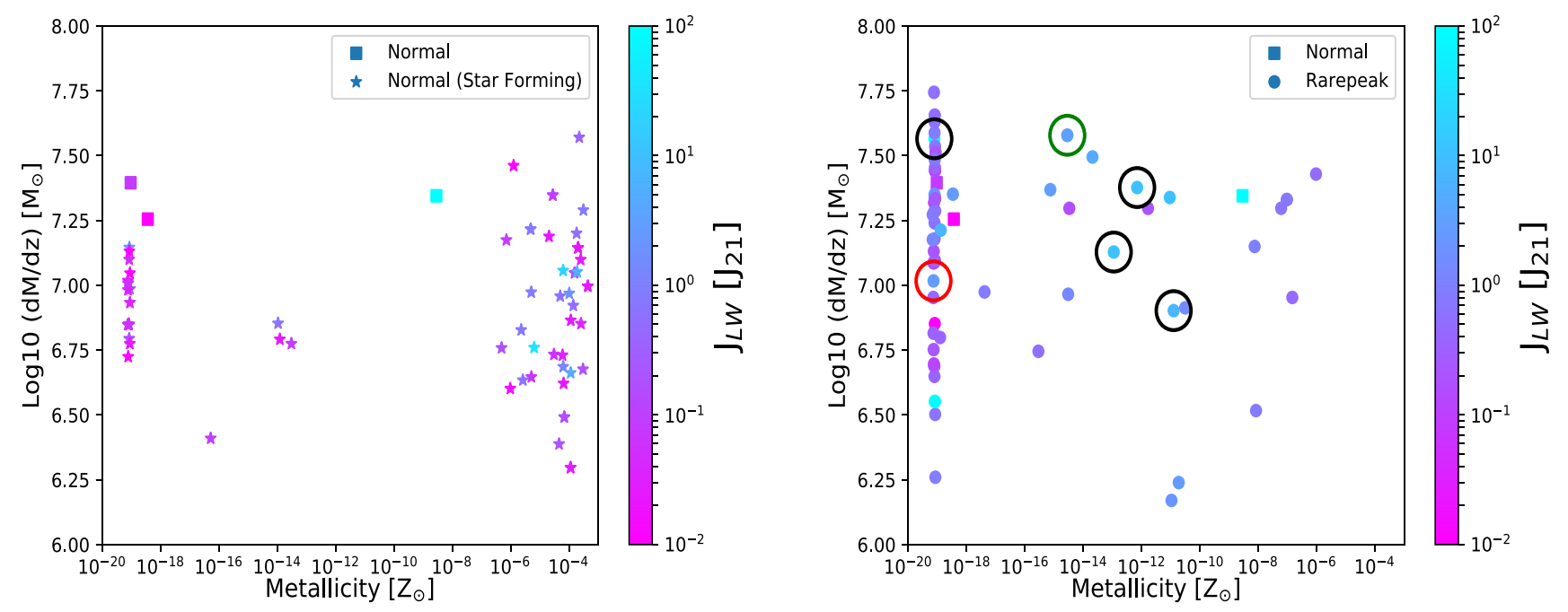

Figure 5. Left-hand panel: Phase space diagram showing the maximum rate of growth (dM/dz) of the DCBH candidate haloes in the Normal region (squares). Also included is the growth rate of a large sample of star-forming haloes for comparison. It should be noted that while the DCBH candidate haloes are among the most rapidly growing haloes, star-forming haloes can grow more rapidly. The colour of the squares, stars, and circles are weighted by the LW radiation to which that halo is exposed prior to the onset of star formation. Right-hand panel: Similar plot for the Rarepeak simulation. The growth rates, $\mathrm{d} M / \mathrm{d} z$, for DCBH candidates in the Rarepeak simulation are shown as circles, again coloured by the level of LW radiation to which they are exposed. The DCBH candidate haloes from the Normal simulations are also plotted for direct comparison. The black outer circles are used to identify four DCBH candidates, which collapse completely isothermally at $T=8000 \mathrm{~K}$. The DCBH candidate halo marked with a red outer circle is the MMHalo from W19 while the green outer circle is the LWHalo from W19.

below $10^{6} \mathrm{M}_{\odot}$ signifies the region below which the mass resolution of Renaissance becomes insufficient to confidently model haloes. Generally, we are able to track haloes below this threshold and into the grey region but below $10^{6} \mathrm{M}_{\odot}$ results should be treated with caution. The dashed blue line is the limit above which a halo must grow in order to overwhelm the impact of LW radiation, $M_{\text {min, LW }}$ (Machacek, Bryan \& Abel 2001; O’Shea \& Norman 2008; Crosby et al. 2013, 2016). The dashed red line is the approximate atomic cooling threshold, $M_{\mathrm{atm}}$, at which point cooling due to atomic hydrogen line emission becomes effective. ${ }^{5}$ Focusing first on the Normal region in the left-hand panel, we plot the growth rate of the three DCBH candidate haloes identified in the left-hand panel of Fig. 2. The DCBH candidate haloes are rapid growers but are not necessarily the fastest growing haloes in the Normal region. To emphasize this comparison, we also plot the growth of three rapidly growing haloes that contain stars. We select the three star-forming haloes from the final output of the Normal region but haloes at other redshifts do of course exist, which are rapidly growing and contain stars. In this case, we see that haloes with high $\mathrm{d} M / \mathrm{d} z$. (i.e. the mass as a function of redshift) values can be star free or star forming and hence having a high $\mathrm{d} M / \mathrm{d} z$, does not necessarily discriminate between DCBH halo candidates by itself. Rapidly growing haloes can become metal enriched through external enrichment processes. The enrichment allows the halo interior to cool and to form stars even in the presence of dynamical heating. Therefore, any semi-analytical model or subgrid prescription that uses $\mathrm{d} M / \mathrm{d} z$ alone as a predictor for DCBH candidates will inevitably overestimate the number of candidates.

The right-hand panel of Fig. 4 shows the growth of DCBH candidate haloes from the Rarepeak simulation. There is a much larger number of DCBH candidate haloes in the Rarepeak region

\footnotetext{
${ }^{5}$ Both $M_{\min , \mathrm{LW}}$ and $M_{\text {atm }}$ evolve with redshift although the dependence is
} weak over the range considered here. compared to the Normal region and hence only the DCBH candidate haloes are included in this plot. Again, we see strong evidence of rapid assembly. All of the haloes show evidence of rapid growth between the LW threshold and the atomic cooling limit, which is able to suppress star formation in all of these haloes. The dynamics of each halo are somewhat unique, with some haloes experiencing major mergers that lead to bursts of dynamical heating while others experience more steady but none the less rapid growth. Furthermore, some haloes will be located closer to massive galaxies that expose the haloes to high LW radiation, which in turn impacts the chemothermodynamical characteristics of the halo in question. We now examine the roles that metallicity, rapid growth, and radiation all play in the assembly of a DCBH candidate halo in more detail.

\subsection{Radiation, metallicity, and rapid growth all play a role}

In Fig. 5, we examine quantitatively the $\mathrm{d} M / \mathrm{d} z$ values from haloes in both the Normal and Rarepeak regions. We compare in a 3D representation the average $\mathrm{d} M / \mathrm{dz}, J_{\mathrm{LW}}$, and metallicity of each of the DCBH candidate haloes as well as a subset of star-forming haloes from the Normal region. In the left-hand panel of Fig. 5, we focus on the Normal region. The phase diagram shows the average growth rate, $\mathrm{d} M / \mathrm{d} z$, as a function of halo metallicity. Each symbol is coloured by the level of LW radiation the halo is exposed to. We plot the $\mathrm{d} M / \mathrm{d} z$, metallicity and $J_{\mathrm{LW}}$ values of both DCBH candidate haloes (squares) and star-forming (stars) haloes. The $\mathrm{d} M / \mathrm{d} z$ value is calculated by determining the time taken for a halo to grow from $5 \times 10^{6} \mathrm{M}_{\odot}$ up to the atomic cooling limit $\left(\sim 3 \times 10^{7} \mathrm{M}_{\odot}\right)$. This measures the mean rate at which mass is accumulated by the halo once it crosses the LW threshold (the blue line in Fig. 4) and up to the point it reaches the atomic cooling limit (the red line in Fig. 4). Both the $J_{\mathrm{LW}}$ value and the metallicity are calculated by taking the final value of $J_{\mathrm{LW}}$ and metallicity, respectively, before 
star formation occurs (star formation leads to additional internal LW radiation and metal enrichment, which we cannot disentangle from external effects). The three DCBH candidate haloes have among the highest $\mathrm{d} M / \mathrm{d} z$ values, which goes some way to explaining why these haloes were able to suppress star formation. The dynamical heating impact of rapid growth is given by

$\Gamma_{\mathrm{dyn}}=\alpha M_{\text {halo }}^{-1 / 3} \frac{k_{\mathrm{b}}}{\gamma-1} \frac{\mathrm{d} M_{\text {halo }}}{\mathrm{d} t}$,

where $\Gamma_{\text {dyn }}$ is the dynamical heating rate, $M_{\text {halo }}$ is the halo total mass, and $\alpha$ is a coefficient relating the virial mass and temperature of the halo (Barkana \& Loeb 2001). Two of the haloes are completely metal free while one of the haloes is experiencing some slight external metal enrichment $\left(\sim 2.88 \times 10^{-9} \mathrm{Z}_{\odot}\right)$. However, it is also clear that there are star-forming haloes growing more rapidly than the star-free haloes. This is not surprising. In the case of the halo in the top right of the left-hand panel, this halo became metal enriched early in the halo assembly process. The halo formed a PopIII star but the halo continued to assemble rapidly. In this case, because of the metal enrichment, the dynamical heating due to rapid assembly is negated completely. Therefore, only haloes that remain metal free and grow rapidly can remain star free.

In the right-hand panel of Fig. 5, we plot the same phase plot for the DCBH candidate haloes (circles) in the Rarepeak simulation. Given the large number of DCBH candidate haloes in the Rarepeak region, we do not include star-forming haloes from the Rarepeak region in this plot. We do, however, include the DCBH candidate haloes (squares) from the Normal region for direct comparison. For these DCBH candidate haloes, there is a wide variation in $\log _{10}(\mathrm{~d} M / \mathrm{d} z)$ with values as low as 6.3 and as high as 7.75. Naively, it would be expected that the haloes with low $\mathrm{d} M / \mathrm{d} z$, values and moderate-to-low $J_{\mathrm{LW}}$ values would form stars. However, inspection of individual haloes reveals bursts of rapid assembly, which can result in the suppression of $\mathrm{H}_{2}$ for at least a sound crossing time (see also W19). The average value of $\mathrm{d} M / \mathrm{d} z$, as plotted here, fails to detect the bursts that can suppress star formation, and in many cases those with low average $\mathrm{d} M / \mathrm{d} z$ values have a strong burst of dynamical heating not easily captured by an average value. We will return to this point and the impact this can have on deriving a semi-analytic prescription in Section 4.

In the right-hand panel, we identify six haloes with circles. Four are marked with black circles. These are haloes that we have found show an isothermal collapse up to the maximum resolution of the Renaissance simulations $(\sim 1 \mathrm{pc})$ and are showing no signs of $\mathrm{H}_{2}$ cooling in the core of the halo. Each of the isothermal haloes that we identify here is typically within a few kiloparsec of a star-forming atomic cooling halo but the candidate halo has not yet become either significantly metal enriched or photoevaporated. None the less, the nearby massive galaxies provided a much higher than average (average $\left.J_{\mathrm{LW}} \sim 1 \mathrm{~J}_{21}\right) J_{\mathrm{LW}}$ value. This scenario is similar to the scenario explored by Dijkstra, Ferrara \& Mesinger (2014). We also identify in red the MMHalo in the Rarepeak simulation at $z=$ 15 and the most irradiated halo (green circle) in the simulation at $z$ $=15$. The most massive and most irradiated haloes were previously identified in W19 and investigated in detail.

In Fig. 6, we show the radial profiles of a number of physical quantities for each of the haloes identified by the circles. The blue line is the MMHalo and the green line (LWHalo) is the most irradiated halo. The other haloes are those that show well-defined isothermal collapse profiles. Both the MMHalo and the LWHalo show clear cooling towards the molecular cooling track (bottom left panel). Each of the other haloes has temperatures greater than $8000 \mathrm{~K}$ all the way in to the centre of the halo and so they remain on the cooling atomic cooling track. In the top left panel, we see that both the MMHalo and the LWHalo have higher $\mathrm{H}_{2}$ fractions as expected. All the haloes increase their $\mathrm{H}_{2}$ as the density increases towards the centre of the halo. In the case of the isothermally collapsing haloes, the fraction remains low enough so that cooling remains dominated by atomic cooling. In the top right panel, we plot the enclosed gas mass as a function of radius and in the bottom right panel the instantaneous accretion rate as a function of radius. The accretion rates for each of the haloes are extremely high, with accretion rates above $0.1 \mathrm{M}_{\odot}$ per year at all radii. Accretion rates greater than approximately $0.01 \mathrm{M}_{\odot} \mathrm{yr}^{-1}$ are thought be required for supermassive star formation (e.g. Schleicher et al. 2013; Sakurai et al. 2016). The MMHalo and the LWHalo cool towards the centre of the halo, meaning that fragmentation into a dense cluster of PopIII stars becomes more likely in those cases. The reason that the MMHalo and the LWHalo cool towards the centre is due to their higher $\mathrm{H}_{2}$ fractions compared to the other four haloes. As can be seen in Fig. 5, each of the four selected haloes has systematically higher LW radiation values impinging on to them, resulting in lower $\mathrm{H}_{2}$ fractions. In addition, for the cases where the collapse remains isothermal the degree of fragmentation can be suppressed, with more massive objects likely to form in that case (Regan \& Downes 2018a, b).

\subsection{Synchronized haloes}

Synchronized haloes have been invoked as a means of generating a sufficiently high LW radiation flux to allow the total suppression of $\mathrm{H}_{2}$ in the core of an atomic cooling halo (Dijkstra et al. 2008; Visbal et al. 2014b; Regan et al. 2017). The scenario supposes that two pristine progenitor atomic cooling haloes cross the atomic cooling threshold nearly simultaneously. The suppression of star formation in both the haloes as they assemble eliminates the possibility of either metal enrichment or photoevaporation from one halo to the other. The first halo to cross the atomic cooling threshold suffers catastrophic cooling due to neutral hydrogen line emission cooling and begins to collapse and form stars. The LW radiation from Halo1 irradiates Halo2, thus suppressing $\mathrm{H}_{2}$ in Halo2 and allowing for the formation of a DCBH. We search the Rarepeak region for synchronized pairs matching the above criteria.

We look for pairs of ACHs that remain pristine and devoid of star formation and are separated from each other by less than $1 \mathrm{kpc}$, but are also at a separation of greater than $150 \mathrm{pc}$ as they cross the atomic cooling threshold. We note that this is likely somewhat optimistic given that the region of synchronization is expected to be between approximately 150 and $350 \mathrm{pc}$ for haloes of this size (Regan et al. 2017). Within the Rarepeak region, we find of a total of five pairs of pristine ACHs that fulfil the basic criteria. In Fig. 7, we show a visualization of four of the five haloes that are candidates for synchronized haloes. In each case, the haloes are separated by distances between approximately 200 and $500 \mathrm{pc}$ at the time of crossing the atomic cooling threshold. In all cases, the haloes are still devoid of star formation but at least one of the haloes in the pair forms stars before the next data output. The total mass of the two atomic cooling haloes in each case is above $10^{8} \mathrm{M}_{\odot}$. Given the proximity of the two haloes at this point, it is difficult to estimate the mass of each halo individually.

Visbal et al. (2014a) examined the formation of DCBH from synchronized haloes and estimated their abundances both analytically and through a $\mathrm{N}$-body simulation. To estimate the abundances of 


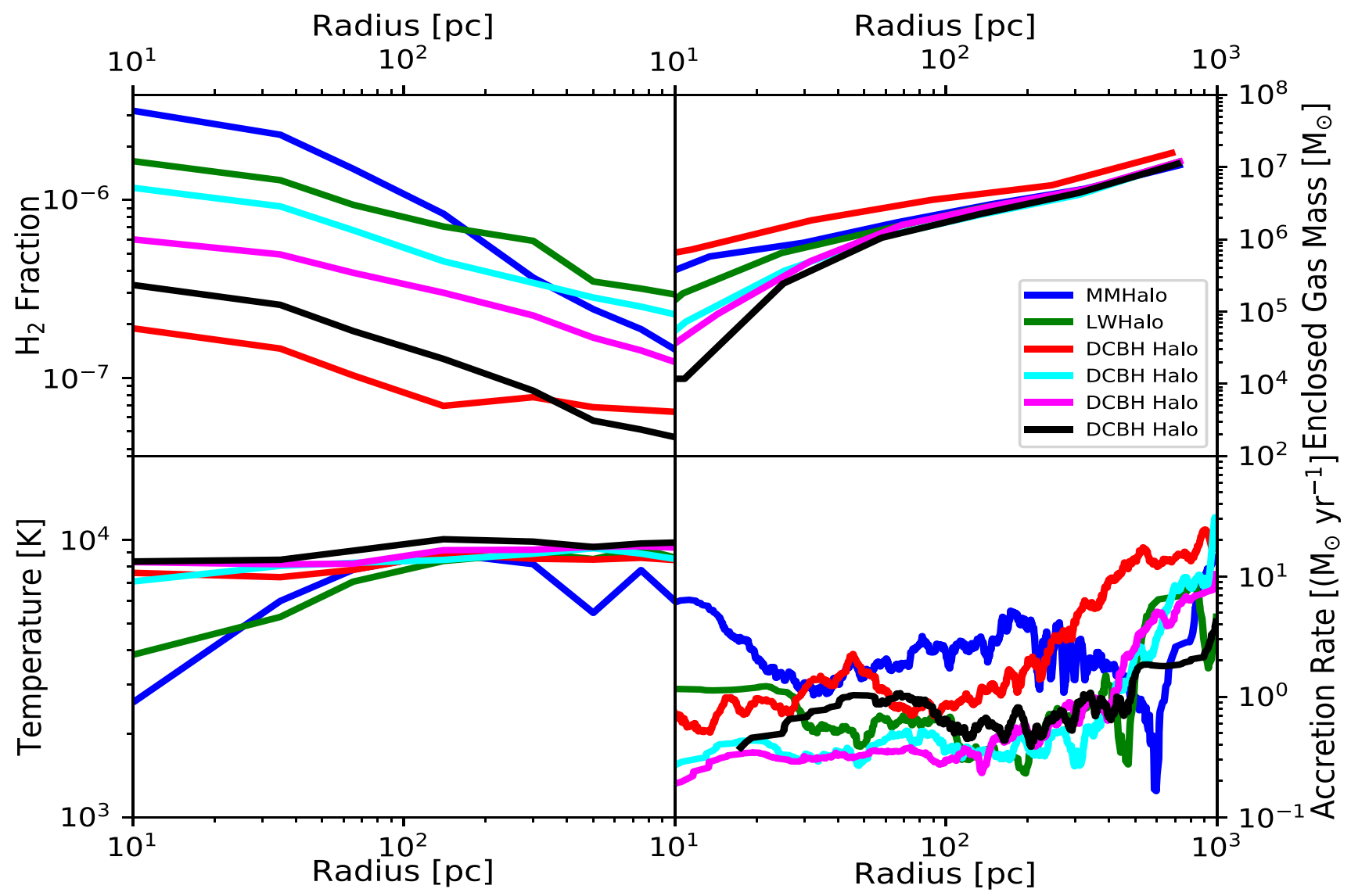

Figure 6. In each of the four panels in this figure, we compare the six DCBH haloes identified in the right-hand panel of Fig. 5. Four of the DCBH candidate haloes are collapsing isothermally while the MMHalo (blue line) and the LWHalo (green line) show strong evidence of a non-isothermal collapse. In the bottom left-hand panel, we plot the temperature against radius, illustrating the isothermality of the four selected DCBH candidate haloes. The MMHalo and the LWHalo clearly start to cool in the halo centre. This cooling can be directly attributed to a higher $\mathrm{H}_{2}$ fraction for the MMHalo and the LWHalo as seen in the top left panel. The enclosed mass for each candidate halo varies inside approximately $30 \mathrm{pc}$ for each halo with an average enclosed mass of $10^{5} \mathrm{M}_{\odot}$ inside $20 \mathrm{pc}$. In the bottom right panel, we show the instantaneous accretion rate for each DCBH candidate halo. All of the haloes show accretion rates greater than $0.1 \mathrm{M}_{\odot} \mathrm{yr}^{-1}$ across several decades in radius and continuing into the core of the halo.

synchronized haloes analytically, they used the following equation:

$\frac{\mathrm{d} n_{\mathrm{DCBH}}}{\mathrm{d} z} \sim \frac{\mathrm{d} n_{\text {cool }}}{\mathrm{d} z}\left(\frac{\mathrm{d} n_{\text {cool }}}{\mathrm{d} z} \Delta_{z_{\mathrm{sync}}} \int^{R . O . R} \mathrm{~d} r 4 \pi r^{2}[1+\eta(r)] f_{\mathrm{s}}(r)\right)$,

where $\frac{\mathrm{d} n_{\text {cool }}}{\mathrm{d} z}$ is the number density of haloes that cross the cooling threshold between $z$ and $z+\mathrm{d} z, \eta(r)$ is the two-point correlation function that describes the enhancement of halo pairs due to clustering, $\Delta_{z_{\text {sync }}}$ is the redshift range corresponding to the synchronization time, and $f_{\mathrm{s}}(r)$ is the fraction of haloes that are found at a radius $r$, when they cross the atomic threshold. Visbal et al. (2014a) used a $N$-body-only simulation to determine the values required for equation (4). They predicted 15 synchronized pairs in a $3375 \mathrm{cMpc}^{3}$ volume. In the Rarepeak region, which has a volume of $133.6 \mathrm{cMpc}^{3}$, we find five synchronized pairs. Given the difference in volume, our abundance is higher by a factor of approximately 5 compared to that of Visbal et al. (2014b). However, the Rarepeak region represents an overdensity of approximately 1.7 compared to an average region of the universe and Visbal et al. (2014a) also performed the calculation at a somewhat lower redshift. When this is taken into account, our values match those of Visbal et al. (2014b) quite well. Furthermore, Visbal et al. (2014b) were unable to account for metal enrichment in their analysis, which may have a led to an overestimate of the number density of synchronized halo candidates in that case.

In order to test the feasibility of the synchronized haloes found in this work, a zoom-in re-simulation of the region surrounding the synchronized pairs is required, which accounts for both normal PopIII star formation, in Halo1, and possible supermassive star formation in Halo2. In order to provide a sufficient flux, Regan et al. (2017) predicted that Halo1 must form approximately $10^{5} \mathrm{M}_{\odot}$ of stellar mass in order to generate a significantly strong LW flux to achieve isothermal collapse. However, the DCBH candidate haloes found here have already had their ability to form $\mathrm{H}_{2}$ suppressed due to dynamical heating. Therefore, these particular haloes may not require such an intense external radiation exposure. A detailed re-simulation of these candidate haloes is now required to quantify the level of LW required in this case.

\section{DISCUSSION AND CONCLUSIONS}

We have analysed the Renaissance suite of highresolution simulations of the early Universe with the goal of identifying candidate haloes in which DCBHs can form. In total, we found 79 haloes over 

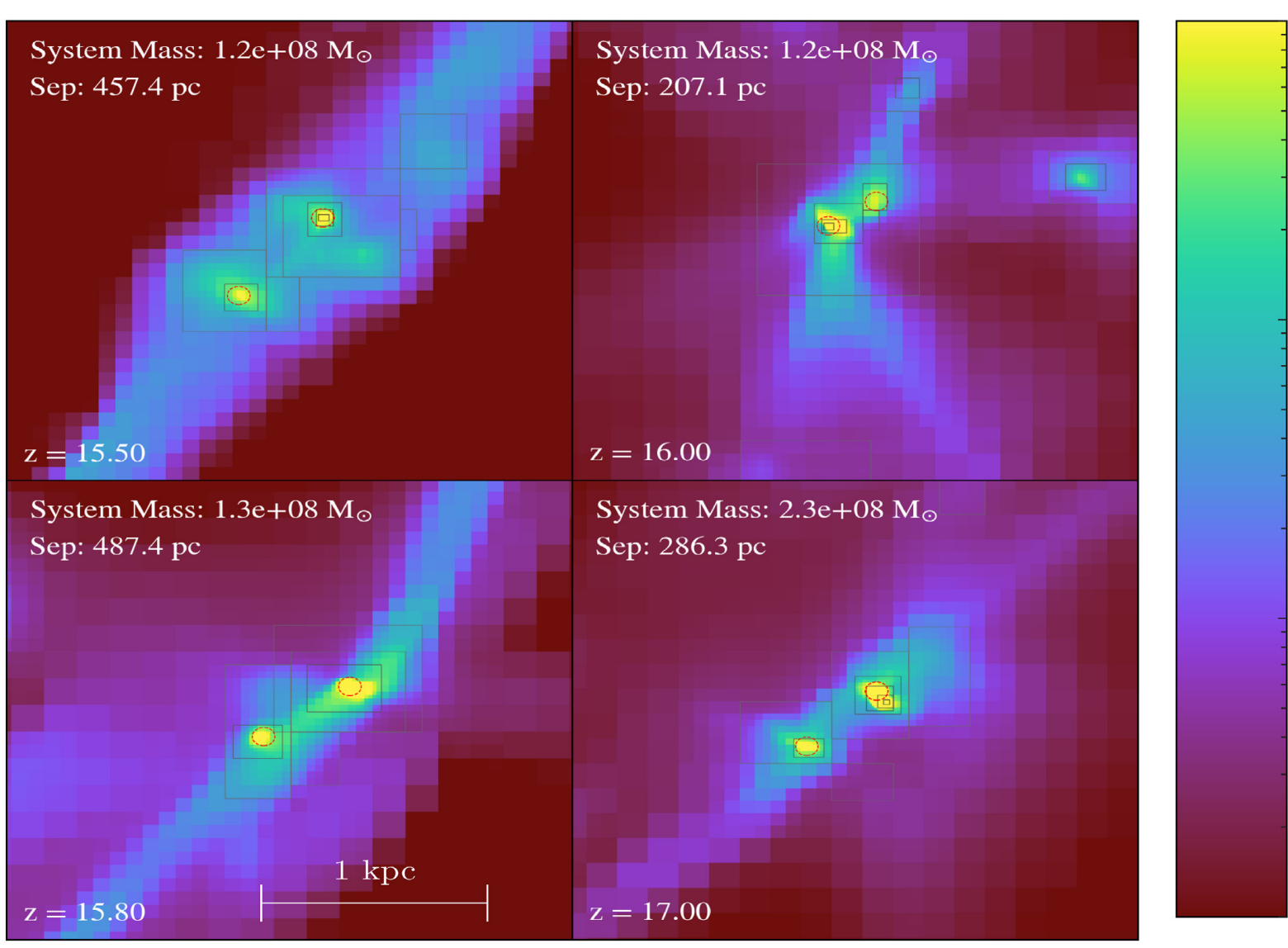

Figure 7. Visualizations of four of the synchronized haloes found in the Rarepeak region. Each member of the synchronized pair is an atomic cooling halo on the cusp of star formation. Typical separations between haloes are between 200 and $500 \mathrm{pc}$ at these outputs. The red circles in each panel mark the central core of each halo. The radius of each circle is approximately 10 per cent of the virial radius. The virial radius of each individual DCBH candidate haloes overlaps with its synchronized partner halo. Only the system mass is shown in each panel since the haloes are subhaloes of each other.

all redshifts and volumes that have crossed the atomic cooling limit and remain both metal free and star free. These 79 haloes represent ideal locations in which to form a DCBH as they will shortly undergo rapid collapse due to neutral hydrogen line emission cooling. The nature of the collapse cannot be probed in these simulations as Renaissance has no subgrid prescription for supermassive star formation and lacks the resolution to accurately track possible fragmentation into a dense stellar cluster of PopIII stars.

In general, the candidate haloes form away from massive galaxies. This allows the candidate haloes to remain free of metal enrichment. In examining the distance that these candidate haloes are from their nearest massive galaxy, we find that the DCBH candidate galaxies typically lie between 10 and $100 \mathrm{kpc}$ from the nearest massive galaxy. These massive galaxies provide $\mathrm{LW}$ intensities that are approximately one order of magnitude higher than the mean intensity expected at these redshifts (Ahn et al. 2015; Xu et al. 2016). However, only a small fraction of the candidate haloes are exposed to LW intensities greater than $10 \mathrm{~J}_{21}$. We find that the primary driver that allows these DCBH haloes to form and remain star free is dynamical heating achieved through the rapid growth of these haloes. The rapid growth is strongly correlated with overdense environments with $76 \mathrm{DCBH}$ candidate haloes forming in the Rarepeak simulation and only $3 \mathrm{DCBH}$ candidate haloes forming in the Normal region. We also note that rapid growth by itself does not guarantee that a halo will become a DCBH candidate. Successfully avoiding metal enrichment must also be accounted for. Hence, in order to derive an accurate subgrid prescription, it will be necessary to account for genetic ${ }^{6}$ metal pollution (Schneider et al. 2006; Dijkstra et al. 2014). We therefore note that only hydrodynamic simulations that self-consistently follow metal transport will be able to successfully identify DCBH candidates in this case. Prescriptions that attempt to identify DCBH candidates only through the rapid growth of (dark matter) haloes will overestimate the number density of DCBH candidates unless a metal enrichment/transport method is also used, which can identify genetic metal enrichment. It should also be noted that sufficient particle (mass) resolution will also be paramount to resolve bursts of accretion, which can delay $\mathrm{H}_{2}$ formation for at least a sound crossing time (W19).

While less than 5 per cent of DCBH candidate haloes are exposed to $\mathrm{LW}$ intensities of greater than $2 \mathrm{~J}_{21}$, these are none the less the candidate haloes that display a complete isothermal collapse. In the vast majority of cases, our examination of the radial profiles of these DCBH candidate haloes shows that the central core of the haloes cools due to the $\mathrm{H}_{2}$. The haloes that collapse isothermally are stronger candidates for forming a supermassive star, while those that collapse non-isothermally and still display rapid inflow are more likely to form a dense stellar cluster (Freitag et al. 2006; Freitag

${ }^{6}$ Genetic metal pollution was initially coined by Dijkstra et al. (2014) and refers to the transfer of metals from smaller to larger haloes via mergers and accretion. 
2008; Lupi et al. 2014; Katz et al. 2015). However, it should be noted that the resolution and subgrid physics modules of Renaissance are not sufficient to probe the further evolution of these haloes. The formation of a supermassive star, a normal population of metal-free stars, and/or a dense stellar cluster may be the final outcome. In order to fully understand the further evolution of these systems, we are now running zoom-in simulations across a handful of interesting haloes in order to undercover the next stage of evolution of these haloes.

Finally, our analysis also reveals the existence of five synchronous haloes with separations between 200 and 500 pc on the cusp of undergoing collapse. These haloes represent excellent candidates for further investigation of the synchronized pair scenario (Dijkstra et al. 2008; Visbal et al. 2014a; Regan et al. 2017). Imminent star formation in one of the haloes will result in the adjacent haloes being subject to intense LW radiation, which will prevent the adjacent halo from cooling due to $\mathrm{H}_{2}$. In that case, the adjacent halo will remain on the atomic cooling track and will be a strong candidate for supermassive star formation. In addition to this, the subsequent merger of the two haloes should provide a plentiful supply of baryonic matter with which to successfully generate a massive black hole seed. Zoom-in simulations of a number of promising DCBH candidate haloes are now underway.

\section{ACKNOWLEDGEMENTS}

JHW was supported by NSF awards AST-1614333 and OAC1835213, NASA grant NNX17AG23G, and Hubble theory grant HST-AR-14326. BWO was supported in part by NSF awards PHY-1430152, AST-1514700, and OAC-1835213, by NASA grants NNX12AC98G and NNX15AP39G, and by Hubble theory grants HST-AR-13261.01-A and HST-AR-14315.001-A. MLN was supported by NSF grants AST-1109243, AST-1615858, and OAC1835213. The Renaissance simulations were performed on Blue Waters operated by the National Center for Supercomputing Applications (NCSA) with PRAC allocation support by the NSF (awards ACI-0832662, ACI-1238993, and ACI-1514580). This research is part of the Blue Waters sustained-petascale computing project, which is supported by the NSF (awards OCI-0725070 and ACI1238993) and the state of Illinois. Blue Waters is a joint effort of the University of Illinois at Urbana-Champaign and its NCSA. We thank an anonymous referee whose comments greatly improved the clarity of the manuscript. The freely available astrophysical analysis code yt (Turk et al. 2011) and plotting library MATPLOTLIB were used to construct numerous plots within this paper. Computations described in this work were performed using the publicly available ENZO code, which is the product of a collaborative effort of many independent scientists from numerous institutions around the world.

\section{REFERENCES}

Abel T., Anninos P., Zhang Y., Norman M. L., 1997, New Astron., 2, 181

Abel T., Bryan G. L., Norman M. L., 2000, ApJ, 540, 39

Abel T., Bryan G. L., Norman M. L., 2002, Science, 295, 93

Agarwal B., Khochfar S., 2015, MNRAS, 446, 160

Ahn K., Shapiro P. R., Iliev I. T., Mellema G., Pen U. L., 2009, ApJ, 695, 1430

Ahn K., Xu H., Norman M. L., Alvarez M. A., Wise J. H., 2015, ApJ, 802, 8

Alexander T., Natarajan P., 2014, Science, 345, 1330

Alvarez M. A., Wise J. H., Abel T., 2009, ApJ, 701, L133

Anninos P., Zhang Y., Abel T., Norman M. L., 1997, New Astron., 2, 209

Bañados E. et al., 2018, Nature, 553, 473
Barkana R., Loeb A., 2001, Phys. Rep., 349, 125

Begelman M. C., Rees M. J., 1978, MNRAS, 185, 847

Begelman M. C., Volonteri M., Rees M. J., 2006, MNRAS, 370, 289

Begelman M. C., Rossi E. M., Armitage P. J., 2008, MNRAS, 387, 1649

Bromm V., Loeb A., 2003, ApJ, 596, 34

Bromm V., Coppi P. S., Larson R. B., 2002, ApJ, 564, 23

Bryan G. L., Norman M. L., O'Shea B. W., Abel T., Wise J. H., Turk M. J., The Enzo Collaboration, 2014, ApJS, 211, 19

Chen P., Wise J. H., Norman M. L., Xu H., O’Shea B. W., 2014, ApJ, 795, 144

Crosby B. D., O’Shea B. W., Smith B. D., Turk M. J., Hahn O., 2013, ApJ, 773, 108

Crosby B. D., O'Shea B. W., Beers T. C., Tumlinson J., 2016, ApJ, 820, 71

Devecchi B., Volonteri M., 2009, ApJ, 694, 302

Dijkstra M., Haiman Z., Mesinger A., Wyithe J. S. B., 2008, MNRAS, 391, 1961

Dijkstra M., Ferrara A., Mesinger A., 2014, MNRAS, 442, 2036

Eisenstein D. J., Hu W., 1999, ApJ, 511, 5

Eisenstein D. J., Loeb A., 1995, ApJ, 443, 11

Fan X., Carilli C. L., Keating B., 2006, ARA\&A, 44, 415

Fernandez R., Bryan G. L., Haiman Z., Li M., 2014, MNRAS, 439, 3798

Field G. B., Somerville W. B., Dressler K., 1966, ARA\&A, 4, 207

Freitag M., 2008, in Beuther H., Linz H., Henning T., eds, ASP Conf. Ser. Vol. 387, Massive Star Formation: Observations Confront Theory. Astron. Soc. Pac., San Francisco, p. 247

Freitag M., Gürkan M. A., Rasio F. A., 2006, MNRAS, 368, 141

Habouzit M., Volonteri M., Latif M., Dubois Y., Peirani S., 2016, MNRAS, 463,529

Haemmerlé L., Woods T. E., Klessen R. S., Heger A., Whalen D. J., 2018a, MNRAS, 474, 2757

Haemmerlé L., Woods T. E., Klessen R. S., Heger A., Whalen D. J., 2018b, ApJ, 853, L3

Hahn O., Abel T., 2011, MNRAS, 415, 2101

Hallman E. J., O’Shea B. W., Burns J. O., Norman M. L., Harkness R., Wagner R., 2007, ApJ, 671, 27

Hosokawa T., Yorke H. W., Inayoshi K., Omukai K., Yoshida N., 2013, ApJ, 778,178

Inayoshi K., Tanaka T. L., 2015, MNRAS, 450, 4350

Inayoshi K., Omukai K., Tasker E., 2014, MNRAS, 445, L109

Inayoshi K., Haiman Z., Ostriker J. P., 2016, MNRAS, 459, 3738

Jenkins A., Frenk C. S., White S. D. M., Colberg J. M., Cole S., Evrard A. E., Couchman H. M. P., Yoshida N., 2001, MNRAS, 321, 372

Katz H., Sijacki D., Haehnelt M. G., 2015, MNRAS, 451, 2352

Latif M. A., Bovino S., Van Borm C., Grassi T., Schleicher D. R. G., Spaans M., 2014, MNRAS, 443, 1979

Latif M. A., Bovino S., Grassi T., Schleicher D. R. G., Spaans M., 2015, MNRAS, 446, 3163

Lupi A., Colpi M., Devecchi B., Galanti G., Volonteri M., 2014, MNRAS, 442, 3616

Machacek M. E., Bryan G. L., Abel T., 2001, ApJ, 548, 509

Madau P., Rees M. J., 2001, ApJ, 551, L27

Merritt D., 2009, ApJ, 694, 959

Milosavljević M., Couch S. M., Bromm V., 2009, ApJ, 696, L146

Mortlock D. J. et al., 2011, Nature, 474, 616

Oh S. P., Haiman Z., 2002, ApJ, 569, 558

O'Shea B. W., Norman M. L., 2008, ApJ, 673, 14

O'Shea B. W., Abel T., Whalen D., Norman M. L., 2005, ApJ, 628, L5

O'Shea B. W., Wise J. H., Xu H., Norman M. L., 2015, ApJ, 807, L12

Pacucci F., Natarajan P., Volonteri M., Cappelluti N., Urry C. M., 2017, ApJ, 850, L42

Press W. H., Schechter P., 1974, ApJ, 187, 425

Regan J. A., Downes T. P., 2018a, MNRAS, 475, 4636

Regan J. A., Downes T. P., 2018b, MNRAS, 478, 5037

Regan J. A., Haehnelt M. G., 2009a, MNRAS, 393, 858

Regan J. A., Haehnelt M. G., 2009b, MNRAS, 396, 343

Regan J. A., Johansson P. H., Haehnelt M. G., 2014a, MNRAS, 439, 1160

Regan J. A., Johansson P. H., Wise J. H., 2014b, ApJ, 795, 137

Regan J. A., Johansson P. H., Wise J. H., 2015, MNRAS, 449, 3766 
Regan J. A., Johansson P. H., Wise J. H., 2016, MNRAS, 459, 3377

Regan J. A., Visbal E., Wise J. H., Haiman Z., Johansson P. H., Bryan G. L., 2017, Nat. Astron., 1, 0075

Sakurai Y., Vorobyov E. I., Hosokawa T., Yoshida N., Omukai K., Yorke H. W., 2016, MNRAS, 459, 1137

Schleicher D. R. G., Palla F., Ferrara A., Galli D., Latif M., 2013, A\&A, 558, A59

Schneider R., Salvaterra R., Ferrara A., Ciardi B., 2006, MNRAS, 369, 825

Shang C., Bryan G. L., Haiman Z., 2010, MNRAS, 402, 1249

Shapiro S. L., Teukolsky S. A., 1979, ApJ, 234, L177

Smith B. D., Regan J. A., Downes T. P., Norman M. L., O'Shea B. W., Wise J. H., 2018, MNRAS, 480, 3762

Sugimura K., Omukai K., Inoue A. K., 2014, MNRAS, 445, 544

Turk M. J., Smith B. D., Oishi J. S., Skory S., Skillman S. W., Abel T., Norman M. L., 2011, ApJS, 192, 9

Turk M. J., Oishi J. S., Abel T., Bryan G. L., 2012, ApJ, 745, 154

Visbal E., Haiman Z., Bryan G. L., 2014a, MNRAS, 442, L100

Visbal E., Haiman Z., Bryan G. L., 2014b, MNRAS, 445, 1056

Whalen D., Abel T., Norman M. L., 2004, ApJ, 610, 14

Wise J. H., Abel T., 2011, MNRAS, 414, 3458
Wise J. H., Turk M. J., Abel T., 2008, ApJ, 682, 745

Wise J. H., Turk M. J., Norman M. L., Abel T., 2012, ApJ, 745, 50

Wise J. H., Demchenko V. G., Halicek M. T., Norman M. L., Turk M. J., Abel T., Smith B. D., 2014, MNRAS, 442, 2560

Wise J. H., Regan J. A., O’Shea B. W., Norman M. L., Downes T. P., Xu H., 2019, Nature, 566, 85

Wolcott-Green J., Haiman Z., 2012, MNRAS, 425, L51

Wolcott-Green J., Haiman Z., Bryan G. L., 2011, MNRAS, 418, 838

Woods T. E., Heger A., Whalen D. J., Haemmerlé L., Klessen R. S., 2017, ApJ, 842, L6

Woods T. E. et al., 2018, preprint (arXiv:1810.12310)

Wu X. B. et al., 2015, Nature, 518, 512

Xu H., Wise J. H., Norman M. L., 2013, ApJ, 773, 83

Xu H., Wise J. H., Norman M. L., Ahn K., O’Shea B. W., 2016, ApJ, 833, 84

Yoshida N., Abel T., Hernquist L., Sugiyama N., 2003, ApJ, 592, 645

This paper has been typeset from a $\mathrm{T}_{\mathrm{E}} \mathrm{X} / \mathrm{LT} \mathrm{E} \mathrm{X}$ file prepared by the author. 